\title{
A thermostatted model for a network of energy sources: Analysis on the initial condition
}

\author{
Marco Dalla Via ${ }^{12,+}$, Carlo Bianca ${ }^{12}$, Ikram El Abbassi', and Abdelmoumen Darcherif ${ }^{\prime 2}$ \\ 'ECAM-EPMI, Laboratoire Quartz EA 7393, 13 Boulevard de l'Hautil, 95092 Cergy-Pontoise (France) \\ 'ECAM-EPMI, Laboratoire LR2E, 13 Boulevard de l'Hautil, 95092 Cergy-Pontoise (France)
}

\begin{abstract}
The energy multisource network is a complex system characterized by the interactions between the energy sources. Recently the thermostatted kinetic theory has been proposed for the modelling of a hybrid energy multisource network with storage. The present paper is devoted to the presentation of a thermostatted kinetic theory model for a network composed of a non-renewable and a renewable energy source. The storage system is modelled by introducing an outer force field. In particular the modelling interest is addressed to the analysis on the initial condition of the distribution functions which describe the two energy sources.
\end{abstract}

Keywords. Thermostatted kinetic theory, energy source, storage system, initial condition

\section{Introduction}

Currently a special attention is paid to the modelling of the energy source network, in order to face the increasing energy demand [1,2]. This issue has attracted researchers coming from different fields. Indeed, the inclusion of the renewable energy sources in the power grid is a challenging task which is necessary to realize the so called "energy transition" $[3,4]$. The renewable energy sources are characterised by a certain intermittency $[5,6]$ which needs to be considered in order to optimize the management of the hybrid multisource network. The introduction of storage systems enables facing the low reliability of the renewable intermittent energy sources [7].

During the last few decades, the concept of "smart grid", [8-10] has emerged to depict the features of the modern energy network. In particular, the interactions and the exchange of information between the sources is the fundamental tool with which the network manages to supply the required energy to the users.

Among the different mathematical approaches, that have been considered for the modelling of the energy distribution network, one has: ODE-based models [11, 12], complex networks [13-15] and optimization approaches [16, 17]. In particular, a number of studies have identified the energy network as a complex system $[18,19]$, because of its interconnected character.

Lately, the paper [20] has proposed the thermostatted kinetic theory [21] as modelling tool for the energy multisource network. The thermostatted kinetic theory has already been employed as general paradigm for the modelling of complex systems in biology $[22,23]$ and within the pedestrian dynamics framework [24].
According to the thermostatted kinetic theory approach, one divides the complex system, i.e. the multisource network, into different subsystems, i.e. the energy sources. The sources of energy are represented by distribution functions over the activity variable, i.e. the produced energy value, which expresses the behaviour and the "strategy" of the subsystems. The binary interactions between the sources drive the time evolution of the network which is depicted by nonlinear integro-differential partial equations. The modelling theory also considers the introduction of an outer force field. The action of the force on the energy sources affects the energy supply and enables to model the charging of the storage system. A specific energy quantity is not yielded to the users and it is used to fill the storage system. The outer force field is balanced by introducing a thermostat term which controls the global activation energy and ensures the existence of a nonequilibrium steady state $[25,26]$. The computation of the thermostat term follows the papers $[27,28]$ where the authors use the Gaussian thermostat in the molecular dynamics simulations.

The authors of this work have proposed in [29], and furtherly developed in [30], a thermostatted model for a hybrid power grid. Specifically, the attention has been focused on the sensitivity analysis on the parameters and in the modelling of the time dependent performance of each source of energy (renewable and non-renewable). The present paper deals with a deeper analysis on the initial condition for the aforementioned model. Specifically, different initial conditions are considered in order to enlighten their effect on the evolution over the energy variable of the distribution functions and on the charging of the storage system. The computational

\footnotetext{
* Corresponding author: m.dallavia@ecam-epmi.com
} 
analysis shows that the distribution functions mostly evolve in the neighborhood of the initial condition. Besides, the charging of the storage system is slower if the initial condition is characterized by low values of energy. The contents of this work are exposed by means of three more sections. The second section presents the thermostatted model for the grid composed of one source of non-renewable energy and one source of renewable energy. The third section deals with the analysis on the initial datum of the distribution functions which represent the two sources of energy. Finally, the fourth section proposes some conclusions.

\section{The thermostatted kinetic theory model}

This section presents the thermostatted model for the network of energy sources with storage proposed in [29]. The model is derived within the paradigm described in [21]. The network $\mathcal{N}$ is composed of two sources of energy: a source of non-renewable energy NR and a source of renewable energy R. According to the proposed modelling framework, every single energy source is associated to a quality parameter $u_{i} \in[0,1]$ for $i \in$ $\{1,2\}: u_{1}=1 / 3$ is related to the low quality energy source NR and $u_{2}=2 / 3$ is related to the high quality energy source R. The energy sources are assumed to be two functional subsystems whose description is realized by a vector of distribution functions $\boldsymbol{f}=$ $\left(f_{1}(t, w), f_{2}(t, w)\right)$, where:

$$
f_{i}(t, w):\left[0,+\infty\left[\times D_{w} \rightarrow \mathbb{R}^{+}, \mathrm{i} \in\{1,2\},\right.\right.
$$

and the continuous variable $w \in D_{w} \subset \mathbb{R}^{+}$denotes the value of the furnished energy. Specifically, $f_{i}(t, w) d w$, for $i \in\{1,2\}$, indicates the number of users who are receiving a value of energy $w \in[w, w+d w]$, produced by the energy source $u_{i}$, at time $t$. Bearing all above in mind, $f_{1}(t, w)$ describes the energy production of the source of non-renewable energy $\mathrm{NR}$ and $f_{2}(t, w)$ describes the energy production of the source of renewable energy $R$.

It is assumed that an outer positive force field $\boldsymbol{F}=$ $(F, F) \in\left(\mathbb{R}^{+}\right)^{2}$ acts on the energy sources: This allows the modeling of the charging of the storage. The whole system is moved out of the equilibrium due to the action of the outer force field. Thus, $\boldsymbol{F}$ is balanced by a thermostat term $\alpha[\boldsymbol{f}](t)$. The thermostat aims at controlling the global activation energy of the network and therefore grants the reaching of a nonequilibrium steady state.

The description of the multisource grid at the macroscopic scale is obtained by considering the distribution functions moments. In detail, the global moment of order $(p, q)$ writes as follows:

$$
\begin{gathered}
\mathbb{E}_{(p, q)}^{[\boldsymbol{F}]}[\boldsymbol{f}](t)=\sum_{i=1}^{2} u_{i}^{p} \int_{D_{w}} w^{q} f_{i}(t, w) d w, \\
\forall p, q \in \mathbb{N} .
\end{gathered}
$$

In particular, the moment of order $(0,0) \mathbb{E}_{(0,0)}^{[\boldsymbol{F}]}[\boldsymbol{f}](t)$ denotes the number of users who receive energy. The moment of order $(0,1) \mathbb{E}_{(0,1)}^{[\boldsymbol{F}]}[\boldsymbol{f}](t)$ denotes the average quantity of energy which is yielded to the users. The moment of order $(2,2) \mathbb{E}_{(2,2)}^{[\boldsymbol{F}]}[\boldsymbol{f}](t)$ denotes the global activation energy of the system. In the case $\boldsymbol{F}=\mathbf{0}$, the notation $\mathbb{E}_{(p, q)}^{[\boldsymbol{F}=\mathbf{0}]}[\boldsymbol{f}](t)$ simplifies to $\mathbb{E}_{(p, q)}[\boldsymbol{f}](t)$.

The following master equation balances the inlet and outlet flows into the microscopic volume $[w, w+d w]$ and gives the time derivative of the distribution functions:

$$
\begin{aligned}
\partial_{t} f_{i}(t, w)+F \partial_{w} & \left(\left(1-\alpha_{(2,2)}[\boldsymbol{f}](t) w\right) f_{i}(t, w)\right) \\
& =J_{i}[\boldsymbol{f}](t, w), \quad i \in\{1,2\} .
\end{aligned}
$$

The functional parameter $\alpha_{(2,2)}[\boldsymbol{f}](t)$ refers to the thermostat term employed to control $\mathbb{E}_{(2,2)}^{[\boldsymbol{F}]}[\boldsymbol{f}](t)$, and writes as follows:

$$
\alpha_{(2,2)}[\boldsymbol{f}](t)=\frac{\mathbb{E}_{(2,1)}^{[\boldsymbol{F}]}[\boldsymbol{f}](t)}{\mathbb{E}_{(2,2)}^{[\boldsymbol{F}]}[\boldsymbol{f}](t)}
$$

The reader interested in the formal derivation of the thermostat (4) is addressed to [29].

The functional operator $J_{i}[\boldsymbol{f}](t, w)=G_{i}[\boldsymbol{f}](t, w)-$ $L_{i}[\boldsymbol{f}](t, w)$, for $i \in\{1,2\}$, models the interactions between the energy sources. The operator $J_{i}[\boldsymbol{f}](t, w)$ is divided into the gain term $G_{i}[\boldsymbol{f}](t, w)$ and the loss term $L_{i}[\boldsymbol{f}](t, w)$, which write as follows:

$$
\begin{aligned}
& G_{i}[\boldsymbol{f}](t, w)= \\
& =\sum_{l=1}^{2} \sum_{j=1}^{2} \int_{D_{w}} \eta_{l j}\left(w_{*}, w^{*}\right) \mathcal{B}_{l j}^{i} \mathcal{A}_{l j}\left(w_{*}, w^{*} ; w\right) \\
& \times f_{l}\left(t, w_{*}\right) f_{j}\left(t, w^{*}\right) d w_{*} d w^{*}, \mathrm{i} \in\{1,2\}, \\
& L_{i}[\boldsymbol{f}](t, w)= \\
& =f_{i}(t, w) \sum_{j=1}^{2} \int_{D_{w}} \eta_{i j}\left(w, w^{*}\right) f_{j}\left(t, w^{*}\right) d w^{*}, \\
& \mathrm{i} \in\{1,2\} .
\end{aligned}
$$

The functional parameters model the phenomena described below:

- $\eta_{l j}\left(w_{*}, w^{*}\right): D_{w} \times D_{w} \rightarrow \mathbb{R}^{+}$, for $l, j \in\{1,2\}$, models the rate of the exchanges of information between the energy source $u_{l}$, yielding the value of energy $w_{*}$, and the energy source $u_{j}$, yielding the value of energy $w^{*}$. The interaction rate is constant. It is assumed that the two distinct energy sources have an interaction rate which is smaller than the autointeraction rate: $\eta_{l j}\left(w_{*}, w^{*}\right)=\alpha+\beta \delta_{l j}$, with $\alpha, \beta \in \mathbb{R}^{+}$, where $\delta_{l j}$ refers to the delta of Kronecker.

- $\mathcal{A}_{l j}\left(w_{*}, w^{*} ; w\right): D_{w} \times D_{w} \times D_{w} \rightarrow \mathbb{R}^{+}$, for $l, j \in$ $\{1,2\}$, represents the probability that the binary interaction involving the energy sources $u_{l}$ and $u_{j}$, which yield the values of energy $w_{*}$ and $w_{*}$, respectively, makes the energy source $u_{l}$ to yield the value of energy $w$. The modeling of $\mathcal{A}_{l j}\left(w_{*}, w^{*} ; w\right)$ is based on the following statements: The low values of energy should be furnished by the non-renewable energy source NR and the high values of energy should 
be furnished by the renewable energy source R. Therefore, the probability density $\mathcal{A}_{l j}\left(w_{*}, w^{*} ; w\right)$ reads:

$$
\mathcal{A}_{l j}\left(w_{*}, w^{*} ; w\right)=\delta\left(\mathrm{w}-d_{l j}\left(w_{*}, w^{*}\right)\right)
$$

where $\delta$ refers to the delta of Dirac and

$$
d_{l j}\left(w_{*}, w^{*}\right)= \begin{cases}w_{*}-\lambda_{1} & \text { if } l=1, j=2 \\ w_{*}+\lambda_{2} & \text { if } l=2, j=1 \\ w_{*} & \text { if } l=j,\end{cases}
$$

and $\lambda_{1}, \lambda_{2} \in[0,1]$. The parameters $\lambda_{1}, \lambda_{2}$ grant the production of different values of energy.

- $\mathcal{B}_{l j}^{i}$, for $i, l, j \in\{1,2\}$, represents the probability that the production of the energy source $u_{i}$ is boosted due to the binary interaction involving the energy sources $u_{l}$ and $u_{j}$. The probability density $\mathcal{B}_{l j}^{i}$ reads:

$$
\mathcal{B}_{l j}^{i}= \begin{cases}\epsilon_{l j} & \text { if } i=l \\ 1-\epsilon_{l j} & \text { if } i \neq l\end{cases}
$$

where $\epsilon_{l j} \in[0,1]$ are the activation parameters. Specifically, the energy source $u_{l}$ has probability $\epsilon_{l j}$ of being activated, for $l, j \in\{1,2\}$.

Given a specified initial datum $\boldsymbol{f}^{\mathbf{0}}=\left(f_{1}^{0}(w), f_{2}^{0}(w)\right)$, the mathematical model (3) is a system of nonlinear integro-differential equations containing 9 phenomenological parameters. The analysis performed in [21] has proved that the corresponding initial boundary value problem has a solution and that this solution is unique. The sensitivity analysis on the parameters has been performed in [29] with special attention to the activation parameters $\epsilon_{12}, \epsilon_{21}$ and the heterogeneity parameters $\lambda_{1}, \lambda_{2}$. Moreover [29] has shown the relation between the magnitude $F$ of the outer force field $\boldsymbol{F}$ and the charging of the storage system. The quantity $\boldsymbol{E}[\mathbf{F}, \mathbf{f}](\mathrm{t})$ denotes the energy placed into the storage system and reads:

$$
\boldsymbol{E}[\mathbf{F}, \mathbf{f}](\mathrm{t})=\mathbb{E}_{(0,1)}[\boldsymbol{f}](t)-\mathbb{E}_{(0,1)}^{[\boldsymbol{F}]}[\boldsymbol{f}](t)
$$

and the equation (2) gives the expression of $\mathbb{E}_{(0,1)}[\boldsymbol{f}](t)$ and $\mathbb{E}_{(0,1)}^{[\boldsymbol{F}]}[\boldsymbol{f}](t)$.

\section{The computational analysis on the initial condition}

This section presents the computational analysis on the initial condition $\boldsymbol{f}^{\mathbf{0}}=\left(f_{1}^{0}(w), f_{2}^{0}(w)\right)$ for the model (3). Specifically, the attention is addressed to the effects of different initial conditions on the evolution over the energy variable of the distribution functions, in the case $F=0$, and on the charging of the storage system, in the case $F \neq 0$.

The parameters presented in the previous section are set as done in [29]: $\alpha=0.6, \beta=0.1, \epsilon_{11}=0.5=\epsilon_{22}, \epsilon_{12}=0.3$, $\epsilon_{21}=0.8, \lambda_{1}=0.5$ and $\lambda_{2}=0.6$. In particular, $\epsilon_{12}<$ $\epsilon_{21}$ boosts the production of the source of renewable energy R, increasing the quality of the energy furnished to the users. The magnitude of the parameters $\lambda_{1}$ and $\lambda_{2}$ has been chosen to promote a diversified production of energy. The sensitivity analysis on the functional parameters can be found in [29].

The numerical integration of the model (3) is performed by means of an order 4 Runge-Kutta method (MATLAB).

The following initial conditions are considered:
a) $f_{i, a}^{0}(w)= \begin{cases}2 / \sqrt{\pi} e^{-(4(w-1.8))^{2}} & \text { if } i=1 \\ 2 / \sqrt{\pi} e^{-(4(w-1.2))^{2}} & \text { if } i=2\end{cases}$
b) $f_{i, b}^{0}(w)= \begin{cases}2 / \sqrt{\pi} e^{-(4(w-2.3))^{2}} \text { if } i=1 \\ 2 / \sqrt{\pi} e^{-(4(w-1.7))^{2}} \text { if } i=2\end{cases}$
c) $f_{i, c}^{0}(w)= \begin{cases}2 / \sqrt{\pi} e^{-(4(w-1.3))^{2}} & \text { if } i=1 \\ 2 / \sqrt{\pi} e^{-(4(w-0.7))^{2}} & \text { if } i=2\end{cases}$

The three initial conditions are shown in the Figures 1, 2 and 3. Each initial condition is defined by means of two Gaussian functions with $\sigma^{2}=1 / 32$. The initial distribution $f_{1, j}^{0}(w)$ attains higher values of $w$ (values of energy) than the initial distribution $f_{2, j}^{0}(w)$, for $j \in$ $\{a, b, c\}$. Accordingly, the three initial conditions are characterized by the fact that the source of nonrenewable energy NR supplies the most part of the energy $\mathbb{E}_{(0,1)}^{[F]}[\boldsymbol{f}](t)$ which is used by the users. For what concerns the number of users, the three initial conditions are such that the two energy sources equally share the total number of users $\mathbb{E}_{(0,0)}^{[\boldsymbol{F}]}[\boldsymbol{f}](t)$. The initial condition a) is characterized by the production of medium values of energy (in the middle of the energy domain $D_{w}$ ), the initial condition b) is characterized by the production of high values of energy (in the right side of the energy domain $D_{w}$ ), the initial condition c) is characterized by the production of low values of energy (in the left side of the energy domain $D_{w}$ ).

\subsection{The $F=0$ case}

This section is devoted to analyzing the evolution over the energy variable of $f_{1}(t, w)$ and $f_{2}(t, w)$, for the initial conditions $\boldsymbol{f}_{\boldsymbol{a}}^{\mathbf{0}}=\left(f_{1, a}^{0}(w), f_{2, a}^{0}(w)\right), \quad \boldsymbol{f}_{\boldsymbol{b}}^{\mathbf{0}}=$ $\left(f_{1, b}^{0}(w), f_{2, b}^{0}(w)\right)$ and $\boldsymbol{f}_{\boldsymbol{c}}^{\mathbf{0}}=\left(f_{1, c}^{0}(w), f_{2, c}^{0}(w)\right)$ in the case $F=0$. As it is shown by the Figures 4, 5 and 6 , the evolution over the energy variable is characterized by the fact that the distribution functions become wider and overlap. This phenomenon is related to the parameters $\lambda_{1}$ and $\lambda_{2}$ which prompt the heterogeneity of the values of the produced energy. The overlapped distribution functions indicate that some users receive a mix of energy produced by both sources. The choice of the magnitude of the parameters $\epsilon_{12}$ and $\epsilon_{21}$, namely $\epsilon_{12}<\epsilon_{21}$, promotes the activation of the source of renewable energy $\mathrm{R}$ whose distribution function $f_{2}(t, w)$ grows more than the distribution function $f_{1}(t, w)$ of the source of non-renewable energy NR. It 
must be stressed that the distribution functions hardly leave the region of the energy domain where the initial condition is located. This means that the energy production is strongly dependent on the initial produced energy values. If at $t^{0}$ the two energy sources are producing low, medium or high values of energy, they will continue to produce low, medium or high values of energy as time passes.

The dependence on the allocation of the initial condition is related to the nature of the kinetic theory framework where the interactions between the subsystems drive the dynamics. Bearing the mathematical model (3) in mind, the intensity of the interactions is proportional to the overlapping between the distribution functions. Therefore, the regions of the energy domain, which are not covered by the distribution functions, are involved by the dynamics only if the distribution functions translate. The translation towards energy values, which are not covered by the initial condition, depends on the magnitude of the parameters $\lambda_{1}$ and $\lambda_{2}$ and promotes a diversified production of energy.

\subsection{The $F \neq 0$ case}

The analysis presented in this section focuses on the energy $\boldsymbol{E}[\boldsymbol{F}, \boldsymbol{f}](t)$, see the equation (10), put into the storage system. The magnitude of the outer force field is set as $F=0.1$. The analysis performed in [29] has shown that, as $F$ increases, more energy $\boldsymbol{E}[\boldsymbol{F}, \boldsymbol{f}](t)$ is put into the storage system. Moreover, it has shown that the thermostatted force field makes the distribution functions shift towards low values of energy. Consequently, the users receive a reduced quantity of energy $\mathbb{E}_{(0,1)}^{[\boldsymbol{F}]}[\boldsymbol{f}](t)$.

As the Figure 7 shows, the charging of the storage system is practically the same with the initial condition $\boldsymbol{f}_{\boldsymbol{a}}^{\mathbf{0}}$ and $\boldsymbol{f}_{\boldsymbol{b}}^{\mathbf{0}}$. At the beginning, the allocation of energy with the initial condition $\boldsymbol{f}_{\boldsymbol{b}}^{\mathbf{0}}$ is slower than the allocation of energy with the initial condition a) and then the former becomes faster than the latter. The initial condition $\boldsymbol{f}_{\boldsymbol{c}}^{\mathbf{0}}$ is characterized by a different augmentation of the energy $\boldsymbol{E}[\boldsymbol{F}, \boldsymbol{f}](t)$ : The allocation of energy into the storage system is much slower than with the other two initial conditions. This is related to the fact that the charging of the storage system begins by taking the high values of energy, see [29]. Therefore, since the initial condition $\boldsymbol{f}_{\boldsymbol{c}}^{\mathbf{0}}$ is characterized by low values of the energy variable and the previous section has shown that the distribution functions evolve in the neighborhood of the initial condition, the action of the outer force field is weakened. It can be now explained why the charging of the storage is not efficient with $\boldsymbol{f}_{\boldsymbol{c}}^{\mathbf{0}}$ : The energy sources provide a little amount of energy to the users and therefore there is a small amount of energy available for the storage system.

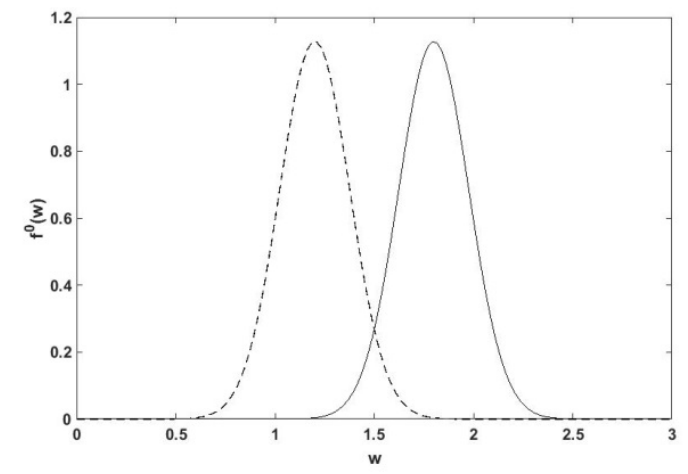

Fig. 1. The initial distribution functions $\boldsymbol{f}_{\boldsymbol{a}}^{\mathbf{0}}$. The solid line refers to the energy source NR and the dashed line refers to the energy source R.

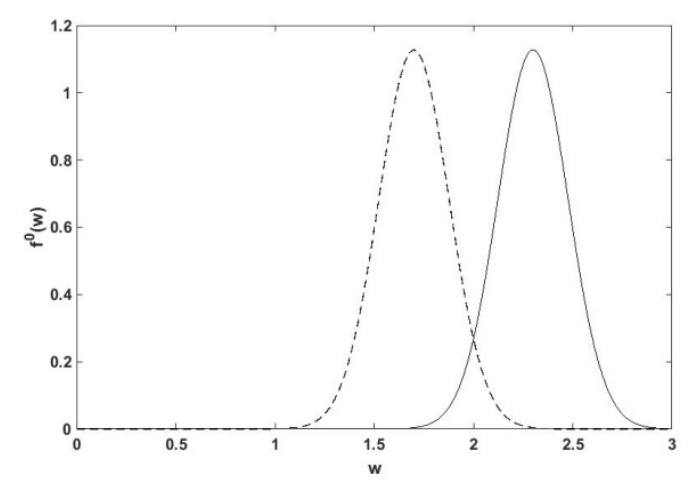

Fig. 2. The initial distribution functions $\boldsymbol{f}_{\boldsymbol{b}}^{\mathbf{0}}$. The solid line refers to the energy source NR and the dashed line refers to the energy source R.

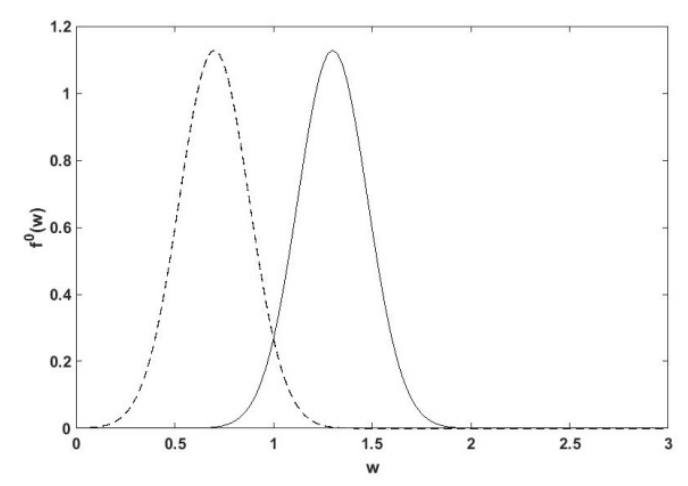

Fig. 3. The initial distribution functions for the case $\boldsymbol{f}_{\boldsymbol{c}}^{\mathbf{0}}$. The solid line refers to the energy source NR and the dashed line refers to the energy source $\mathrm{R}$. 


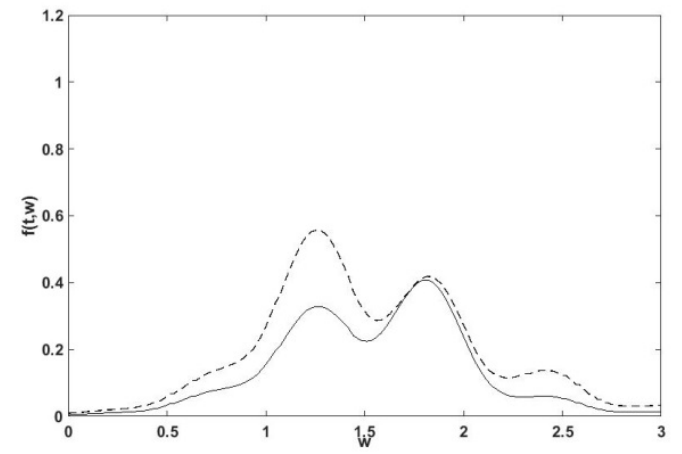

Fig. 4. The evolution over the energy variable of the distribution functions at time $t=30$ for the initial datum $\boldsymbol{f}_{\boldsymbol{a}}^{\mathbf{0}}$. The solid line refers to the energy source NR and the dashed line refers to the energy source R.

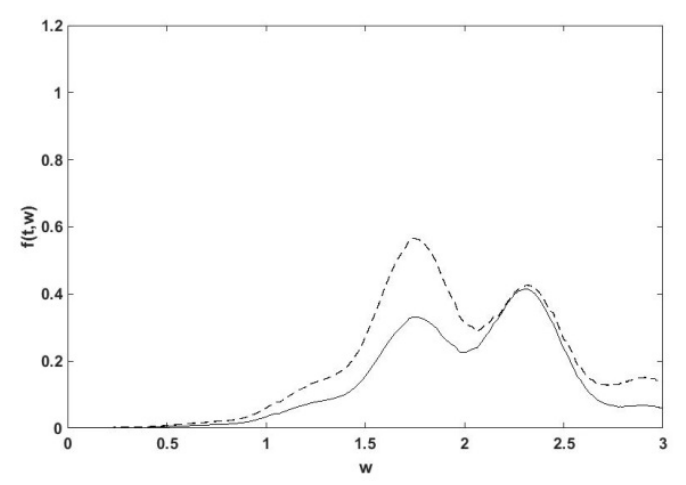

Fig. 5. The evolution over the energy variable of the distribution functions at time $t=30$ for the initial datum $\boldsymbol{f}_{\boldsymbol{b}}^{\mathbf{0}}$. The solid line refers to the energy source NR and the dashed line refers to the energy source R.

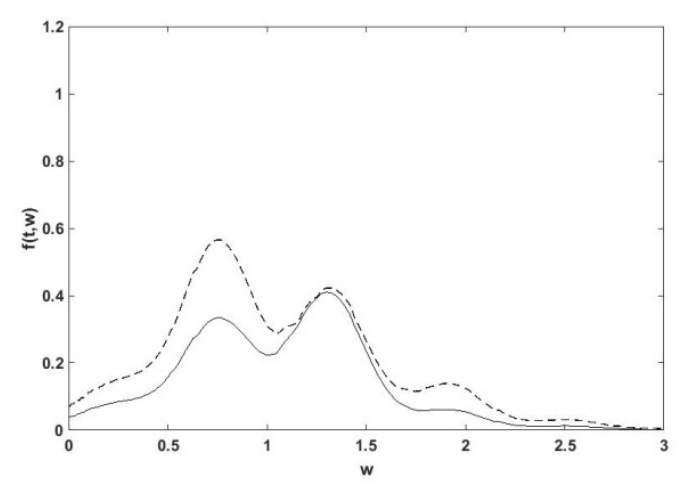

Fig. 6. The evolution over the energy variable of the distribution functions at time $t=30$ for the initial datum $\boldsymbol{f}_{\boldsymbol{c}}^{\mathbf{0}}$. The solid line refers to the energy source NR and the dashed line refers to the energy source $\mathrm{R}$.

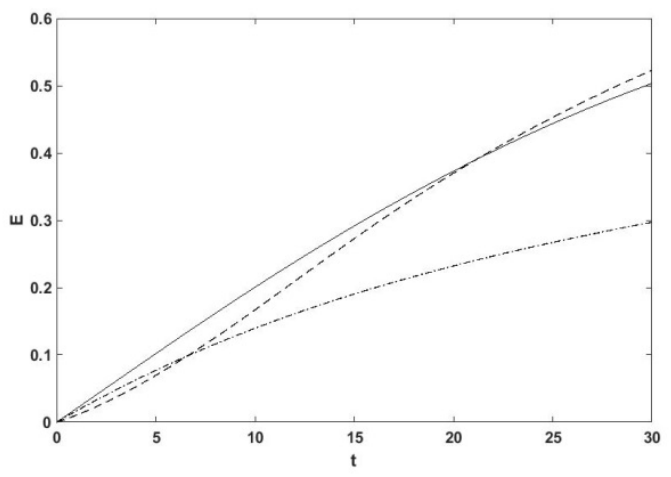

Fig. 7. The charging of the storage system (energy $\boldsymbol{E}[\boldsymbol{F}, \boldsymbol{f}](t)$ ) for $F=0.1$. The solid line refers to the initial datum $\boldsymbol{f}_{\boldsymbol{a}}^{\mathbf{0}}$, the dashed line refers to the initial datum $\boldsymbol{f}_{\boldsymbol{b}}^{\mathbf{0}}$ and the dashed-dotted line refers to the initial datum $\boldsymbol{f}_{\boldsymbol{c}}^{\mathbf{0}}$.

\section{Conclusions}

This work has performed the analysis on the initial condition for the mathematical model treated in [29]. The model is derived within the thermostatted kinetic theory framework exposed in [21] for the modelling of a grid of hybrid energy sources with storage. According to the mathematical framework, a distribution function $f(t, w)$ describes each source of energy, while an outer force field $\boldsymbol{F}$ models the charging of the storage system. The mathematical model needs a thermostat term $\alpha$ for the purpose of balancing the outer force field and ensure the existence of a nonequilibrium steady state. A system of integro-differential equations depicts the time derivative of the distribution functions.

In particular, the present work has considered a source of non-renewable energy and a source of renewable energy. The authors have investigated the effects of the initial condition on the way the distribution functions evolve in the energy variable and on the charging of the storage system. Three different initial conditions have been considered, characterized by medium, high and low values of the energy variable $w$. In the case $\boldsymbol{F}=\mathbf{0}$, the computational analysis has shown that the distribution functions evolve in the neighbourhood of the initial condition because of the interaction-driven dynamics of the multisource grid. In the case $\boldsymbol{F} \neq \mathbf{0}$, the computational analysis has shown that, if the initial condition is characterized by low values of energy, the charging of the storage system is slower.

The analysis performed in this paper can be generalized: Different initial conditions can be considered, characterized by a different shape and by a different sharing of the total number of users, i.e. $\mathbb{E}_{(0,0)}^{[\boldsymbol{F}]}[\boldsymbol{f}](t)$, and of the average quantity of energy supplied to them, i.e. $\mathbb{E}_{(0,1)}^{[\boldsymbol{F}]}[\boldsymbol{f}](t)$, between the energy sources. Besides the analysis on the initial datum can be performed for the model presented in [30] where the authors have introduced time dependent activation parameters. 


\section{References}

1. S. Jebaraj, S. Iniyan, Renew. Sust. Energ. Rev. 10, 218311 (2006)

2. P.H.J. Nardelli, N. Rubido, C. Wang, M.S. Baptista, C. Pomalaza-Raez, P. Cardieri, M. Latva-aho, Eur. Phys. J. Special Topics 223, 2423-2437 (2014)

3. B.D. Solomon, K. Krishna, Energy Policy 39, 74227431 (2011)

4. A.S. Dagoumas, N.E. Koltsaklis, Appl. Energy 242, 1573-1587 (2019)

5. G. Notton, M.-L. Nivet, C. Voyant, C. Paoli, C. Darras, F. Motte, A. Fouilloy, Renew. Sust. Energ. Rev. 87, 96105 (2018)

6. F. Romanelli, Eur. Phys. J. Plus 131, 53 (2016)

7.Y. Yang, S. Bremner, C. Menicats, M. Kay, Renew. Sust. Energ. Rev. 91, 109-125 (2018)

8. S. Blumsack, A. Fernandez, Energy 37, 61-68 (2012)

9. X. Fang, S. Misra, G. Xue, D. Yang, IEEE Commun. Surv. Tutor. 14, 944-980 (2012)

10. M. Ourahou, W. Ayrir, B. EL Hassouni, A. Haddi, Math. Comput. Simulation https://doi.org/10.1016/j.matcom.2018.11.009

11. G. Filatrella, A.H. Nielsen, N.F. Pedersen, Eur. Phys. J. B 61, 485-491 (2008)

12. R. Carareto, M.S. Baptista, C. Grebogi, Comm. Nonlinear Sci. Numer. Simulat. 18, 1035-1046 (2013)

13. A. Sciré, I. Tuval, V.M. Eguíluz, Europhys. Lett. 71, 318-324 (2005)

14. G.A. Pagani, M. Aiello, Physica A 392, 2688-2700 (2013)

15. B.C. Wang, M. Sechilariu, F. Locment, Math. Comput. Simulation 91, 119-133 (2013)

16. R. Baños, F. Manzano-Agugliaro, F.G. Montoya, C. Gil, A. Alcayde, J. Gómez, Renew. Sust. Energ. Rev. 15, 1753-1766 (2011)

17. K.-H. Chang, G. Lin, Simul. Model. Pract. Theory 52, 40-51 (2015)

18. S. Mei, X. Zhang, M. Cao, Power Grid Complexity, (Springer, 2011)

19. C. Peterman, S.B. Amor, A. Bui, Advances in Knowledge-Based and Intelligent Information and Engineering Systems, 149-158, (Frontiers in Artificial Intelligence and Applications, IOS Press, 2012)

20. M. Dalla Via, C. Bianca, I. El Abbassi, A.-M. Darcherif, A hybrid thermostatted kinetic framework for the modeling of a hybrid multisource system with storage, submitted (2018)

21. C. Bianca, Appl. Math. Sci. 6, 651-660

22. C. Bianca, J. Riposo, Eur. Phys. J. Plus 130, 159 (2015)

23. C. Bianca, L. Brézin, Int. J. Biomath. 10, 1750072 (2017)

24. C. Bianca, C. Mogno, Eur. Phys. J. Plus 133, 213 (2018)
25. G.P. Morriss, C.P. Dettmann, Chaos 8, 321-336 (1998)

26. O.G. Jepps, L. Rondoni, J. Phys. A 43, 133001 (2010)

27. W.G. Hoover, A.J.C. Ladd, B. Moran, Phys. Rev. Lett. 48, 1818-1820 (1982)

28. D.J. Evans, W.G. Hoover, B.H. Failor, B. Moran, Phys. Rev. A 28, 1016-1021 (1983)

29. M. Dalla Via, C. Bianca, I. El Abbassi, A.-M. Darcherif, Appl. Math. Model. 78, 232-248 (2020)

30. M. Dalla Via, C. Bianca, I. El Abbassi, A.-M. Darcherif, Eur. Phys. J. Plus 135, 198 (2020) 